Results 492 CA19.9 assays were performed in 12 months. 245 were in patients who had not had abdominal imaging or LFTs measured. 247/492 had both imaging and LFTs and were included in the initial analysis. 102/247 had a positive CA19.9. A total of 45/247 were found to have a pancreatic or bilary malignancy $38 / 45$ had a positive CA19.9. This was negative in $7 / 45$. The overall clinical utility of CA19.9 was poor. From the 492 assays performed during the year a positive CA19.9 was associated with a new malignancy in just $7 \%$ (38/492). Overall $63 \%$ of patients with a positive CA19.9 did not have pancreatic cancer (PPV 37\%) Conversely $5 \%$ of those with a negative result did ( $95 \% \mathrm{NPV}) .22 \%$ of patients with pancreatic malignancy had normal LFTs at the time of diagnosis. In total 103/ $492(20 \%)$ of assays were duplicates. Only $36 / 103$ (35\%) were in patients with confirmed malignancy. 67 assays were requested in patients with normal abdominal imaging or in the absence of imaging and LFTs. The total cost for all assays was $£ 7380$. $£ 6165$ was spent on inappropriate requests or where the diagnosis was not pancreatic malignancy.

Conclusion The clinical utility of CA19.9 is poor with only $7 \%$ of samples resulting in a new diagnosis of pancreatic malignancy. The assay is frequently requested inappropriately, often without abdominal imaging being available. LFTS should not be used to guide testing. CA19.9 measurement should only be undertaken in patients where imaging results are available and suggestive of pancreatic malignancy. There are significant cost savings from this approach.

Competing interests None declared.

\section{PTU-253 LIFE WITHOUT A BLEEDING ROTA: RISK OF LATE ENDOSCOPY?}

doi:10.1136/gutjnl-2012-302514c.253

R Dart, ${ }^{*}$ D Dewar. Department of Gastroenterology, University Hospital Lewisham, London, UK

Introduction In the 2007 UK audit of acute upper gastrointestinal bleeding (AUGIB), ${ }^{1} 44 \%$ of participant centres did not have facility for a dedicated bleeding rota. Gross measures of outcome, such as mortality, were found to be independent of the presence of a bleeding rota; however adherence to other aspects of guidance, such as time to endoscopy, in the absence of a bleeding rota, was not examined. We aimed to examine the performance in the absence of a dedicated bleeding rota, but with a "goodwill" out of hours service, against national guidelines and performance.

Methods Data were collected prospectively for all cases of AUGIB over a 2-month period from September 2010 to November 2010. Cases were identified from request forms in the endoscopy department. Data were collected for all endoscopies; repeat endoscopy on the same admission was recorded separately. Rockall score, if not included with the original endoscopy request was calculated retrospectively. Results were analysed in Excel. Statistics performed using Fischer's exact test.

Results There were 35 patients identified, referred for 40 procedures for AUGIB, 39 of which were carried out. Median age at presentation was 64 (35-92), 14 years younger than the national audit. The proportion of patients presenting with documented cirrhosis $23 \%$ $(n=8)$ was significantly greater than in the national audit $p=0.0026$. Furthermore the proportion of patients with varices, although not necessarily responsible for the bleed, was greater $17 \%$ vs $8 \% p=N S$. Rockall score was documented in $23 \%$ cases compared with $19 \%$ in the national audit. Rockall score was $\geq 3$ in $73 \%$ compared with $51 \%$ in the national audit $p=0.01$. Time to endoscopy, regardless of Rockall score was $<24 \mathrm{~h}$ in $72 \%$ and in patients with Rockall score $\geq 3$ was $79 \%$ compared with $50 \%$ in both groups in the national audit. In the cases in our study for whom the Rockall score was documented, all had a Rockall score $\geq 3$ and proceeded to endoscopy within $24 \mathrm{~h}$. Mortality in both our study and the national audit was $10 \%$.

Conclusion Our patient group was younger than the national average with a higher proportion with pre-endoscopy Rockall score $\geq 3$. We demonstrate ongoing difficulties in encouraging documentation of risk assessment scores, however in our experience when the Rockall score was documented the endoscopy happened in a timely fashion. We demonstrate with data from our centre that early endoscopy is achievable in the majority of cases in the absence of a dedicated bleeding rota. This does not appear to affect overall mortality however risk assessment and early endoscopy are important as we strive to improve the service we offer our patients.

Competing interests None declared.

\section{REFERENCE}

1. UK Comparative Audit of Upper Gastrointestinal Bleeding and the Use of Blood. London: British Society of Gastroenterology, 2007. http://www.bsg.org.uk/ pdf word docs/blood audit report 07.pdf

\section{PTU-254 WHAT DO GENERAL PRACTITIONERS WANT FROM GASTROENTEROLOGY SERVICES IN SECONDARY CARE, AND WHAT ARE THEY HAPPY MANAGING IN PRIMARY CARE?}

doi:10.1136/gutjnl-2012-302514c.254

R Basuroy, ${ }^{*}$ J Ramage, M Reynolds. Department of Gastroenterology, Basingstoke and North Hampshire Hospital, Hampshire Hospitals NHS Trust, Basingstoke, UK

Introduction Recent changes in the NHS have focussed on moving more services into primary care. General Practitioners (GPs) are rarely consulted over planning of secondary care services, nor are they asked on their own comfort in managing gastroenterological conditions. The results of a GP survey of confidence and service development are presented.

Methods An online survey (created on Google Docs) was emailed to 102 GP practices. GPs were asked to rate their confidence in managing gastroenterological conditions as well as rate service developments using a 5-point Likert scale (1-very low, 5-very high). Free text boxes allowed GPs to provide additional comments for qualitative analysis. Responses $(n=52)$ within 28 days were analysed in Microsoft Excel.

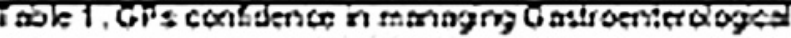
constions

\begin{tabular}{|c|c|c|}
\hline Condiditiven & sivesoge & Predar \\
\hline Orsp'wg & 8.00 & 8 \\
\hline GORO axd 0,repta & $\therefore .00$ & \\
\hline ien Do (ciency Ander is & $\therefore .00$ & \\
\hline Gat Sord ard Basy Disesto & 3.93 & \\
\hline 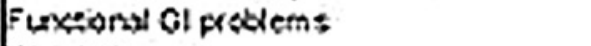 & 3.87 & \\
\hline Acighe los: & 3.78 & 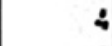 \\
\hline Lize and Cryoric D istlyces & 3.75 & \\
\hline 2odanimalp ain & 3.79 & $\checkmark$ \\
\hline Oagroreegina malpansies & 358 & ( \\
\hline Lutely DerangedLFTs & 3,35 & . \\
\hline Potierts on Immunosuppre cemts & 3.33 & 4 \\
\hline Iniom rostory Hosel Olsease & 3.33 & 4 \\
\hline Hookd Defensenoy Syndrome & $3.0 S$ & 3 \\
\hline 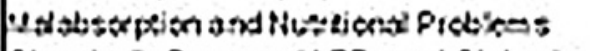 & 3.00 & 3 \\
\hline 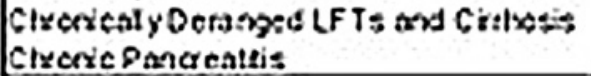 & $\begin{array}{l}2.79 \\
2.26\end{array}$ & 2 \\
\hline
\end{tabular}


Results Abstract PTU-254 table 1 outlines GPs' confidence in managing gastroenterological conditions while Abstract PTU-254 table 2 outlines their rating of service developments in chronic disease management, referral pathways and other services. GPs commented on the need for "clear referral pathways" and "rapid access clinics" outside of the 2-Week Rule remit. They wanted "clear plans" for shared care with "rapid access if problems" as well as "support from nurse specialists" and "access to telephone advice" for both patients and themselves. GPs wanted "workload implications to be recognised" and "money to follow the patient" if more patients are managed in primary care.

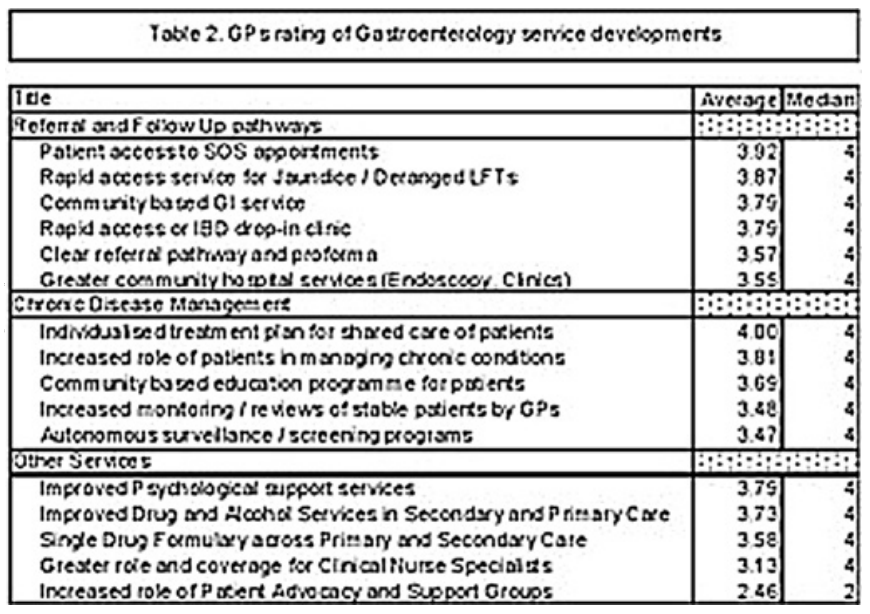

Conclusion The survey has identified which conditions GPs are confident managing in primary care and those which need additional support from secondary care. Future service development is needed in areas of chronic disease management, referrals pathways and allied services. GPs value rapid access to secondary care as well as patient access to SOS appointments and nurse specialists. Developing local pathways, such as with Map of Medicine, can help with referrals and managing chronic conditions in primary care. Online surveys are an easy way to ask GPs about their own confidence in managing gastroenterological conditions as well as their opinion on service developments.

Competing interests None declared.

\section{PTU-255 IS THE SERVICE PROVISION OF DIRECT ACCESS UPPER GI ENDOSCOPY BEING USED EFFECTIVELY BEYOND 2010?}

doi:10.1136/gutjnl-2012-302514c.255

${ }^{1} \mathrm{~S}$ Mahmood, ${ }^{*}$ S S Singh, ${ }^{1} \mathrm{M}$ Naseer, ${ }^{1} \mathrm{~S}$ Sarkar. ${ }^{1}$ Department of Gastroenterology, Royal Liverpool University hospital, Liverpool, UK; ${ }^{2}$ Department of Medicine, Royal Liverpool University hospital, Liverpool, UK

Introduction Upper GI Endoscopy can generate significant income through payment by results (PBR) in the UK. Primary care physicians have a direct access for endoscopy via open access (GPOA) or 2 -week rule (2WR) systems and appropriateness of these lists have been long-debated. With the current financial cut backs within the NHS, it is worthwhile to re-evaluate their effectiveness.

Aim To assess the effectiveness of GPOA/2WR upper GI Endoscopy service.

Methods Retrospective audit of GPOA and 2WR lists between April and October 2011 was performed. 483 referrals were audited $(n=400$ $2 \mathrm{WR}, \mathrm{n}=83 \mathrm{GPOA}$ ) for patient demographics, indications, significant diagnosis, additional tests and patient outcomes.

Results 2WR-Patients: Audit capture rate $86 \%$. Median age of the patient was 65 years (range 18-96), 58\% were Females, with median ASA 2. NICE criteria for referrals was not met in $12 \%$ and a further $14 \%$ had a gastroscopy within 3 years. Outcomes: Significant diagnoses (cancer, oesophagitis, Barretts, PUD, Coeliac Disease, Stricture, Helicobacter pylori gastritis) were present in $44 \%$ (cancer diagnosis $3.4 \%$ ). An endoscopy urease test was performed in $48 \%$, with a $30 \%$ positivity rate. Biopsies were taken in $64 \% .86 \%$ were discharged back to the GP, $4 \%$ were referred to clinic, $6 \%$ booked for a repeat endoscopy and $4 \%$ referred to cancer MDT. GPOA Patients: Audit capture rate was $96 \%$ referrals. Patients were younger than the 2WR with median age 52 years (range 17-86) $(p=0.0001)$, and healthier with median ASA 1 ( $p=0.0001) .53 \%$ were females. $31 \%$ of patients did not meet NICE guidelines and $4 \%$ had a gastroscopy within 3 years. Outcomes: Significant diagnoses were made in $29.9 \%$ (cancer diagnosis $1.2 \%$ ). While the cancer diagnoses with $2 \mathrm{WR}$ was comparable $(\mathrm{p}=0.2)$, there were less benign diagnoses $(p=0.01)$. Additional tests included urease test in $19 \%$, positivity rate $20 \%$ and biopsies $14 \%$. Patient outcomes were similar to 2WR with $88 \%$ of patients discharged directly back to GP, $6 \%$ booked for a repeat endoscopy, 4\% referred to Gastroenterology Clinic and $2 \%$ to a cancer MDT. Overall, 51 patients (12\%) had a previous endoscopy within 3 years, none of which had cancer and 83 patients $(19 \%)$ did not meet NICE guidelines that is, $31 \%$ were inappropriate.

Conclusion With $31 \%$ of patients being inappropriate and low cancer pick up rate, the value of these lists may be questionable. However benign pathology was significant in both lists and therefore process mapping the patient to see if the endoscopy changed the patient's management would be useful given the very high discharge rate to the GP. There still remains significant room for improving the effective use of these services.

Competing interests None declared.

\section{PTU-256 OPTIMISING BOWEL SCREENING COLONOSCOPY UPTAKE WITH NURSE-LED TELEPHONE ASSESSMENT CLINICS}

doi:10.1136/gutjnl-2012-302514c.256

S Osmond. * Aneurin Bevan Health Board, Caerphilly, UK

Introduction Bowel Screening Wales utilises nurse-led telephone assessments pre-colonoscopy for screening participants following a positive faecal occult blood test (FOBt) result. This approach has been shown to optimise colonoscopy uptake.

Methods A literature review was carried out to determine the cost effectiveness and efficiency of telephone assessment as indicated by overall screening colonoscopy appointment uptake. Nurse-led telephone assessment is an advanced practice. Nurses undertaking this form of assessment should have undergone suitable training and supervision, be competent in their practice and accountable for their actions. Advanced assessment is the detailed, systematic collection of relevant information about the patient's problems and health status which requires a specialist knowledge, skill and extensive experience to uncover the relevant information being given and discard the irrelevant. ${ }^{1}$ Bowel Screening Wales was introduced nationally in October 2008 and operates from a single hub which is responsible for inviting participants, processing completed kits and providing results for participants. There are 13 Local Assessment Centres (LAC) which have a responsibility to provide endoscopy, pathology and radiology services for participants who have had a positive FOBt result. Specialist Screening Practitioners are based in each LAC. In Wales each Health Board covers a large geographical area therefore telephone assessments are a more effective method of pre-colonoscopy assessment in terms of cost, time and travel requirements. It has also been found that this approach results in a lower colonoscopy default rate due to improved participant involvement in the decision making process. 